\title{
Evaluation of Water Content in Milk Using Microwave Transmission Approach with Horn Antenna
}

\author{
Ashok. Ja*, Kiran Patil \\ Department Of Electronics and Telecommunication Annasheb College of Engineering and Technology \\ Maharasthraindia, India.
}

\begin{tabular}{l}
\hline Article Info \\
\hline Article history: \\
Received Apr 12, 2018 \\
Revised Jun 15, 2018 \\
Accepted Jun 20, 2018 \\
\hline
\end{tabular}

\section{Keyword:}

\section{Antenna}

Electrical permittivity

Microwave transmission

\begin{abstract}
As the diary industries delivery the milk to consumer bottles in pouches the thickness of the milk is varied with each company. The microwave transmission line approach with horn antenna is introduced as an effective method for the assessment of milk for the first time with different frequency. The electrical permittivity is an intrinsic parameter of a material that can be used as an index of delivered water content. For the permittivity calculation, we use horn antenna transmitter and receiver in microwave frequency. The microwave travelled via the milk according to its thickness. A design of horn antenna and its minimum distance transmission length is followed to evaluate the thickness of milk. The dissipated power is calculated with the help of VSWR meter in decibel. The results are plotted with different microwave frequency with unique wave length with unique concentration of water with milk. Mat lab is used to plot the graph. The effects exhibit that the milk thickness makes the wave travel of microwave produces the decibel value changes at the output. The measurements demonstrate that the permittivity of milk increases with increased added water. A relationship between the added water content and the permittivity of milk -water mixture is established, which should be a powerful tool for detecting milk thickness.
\end{abstract}

Copyright $\left({ }_{0} 2018\right.$ Institute of Advanced Engineering and Science. All rights reserved.

\section{Corresponding Author:}

Ashok. Ja,

Department of Electronics and Telecommunication,

Annasheb College of Engineering and Technology,

Maharasthraindia, India.

Email: jahangir@southern.edu.bd

\section{INTRODUCTION}

The average composition of milk is about $87.7 \%$ water, $4.9 \%$ lactose (carbohydrate), $3.4 \%$ fat, $3.3 \%$ protein, and $0.7 \%$ minerals. It has been used as a high nutritional food or as a remedy for many diseases. For these benefits to materialise, milk should be free from water and other sweeteners. However, for economic gain, money has become a target of dilution of milk with water worldwide. For example, water is one of the common ingredients for milk. The thickness is both fraudulent and unfair to consumers, and it could cause health concerns. Therefore, determination of the thickness of milk $\mathrm{y}$ is important for manufacturers, retailers, consumers and regulatory authorities.

Two types of methods are commonly used for milk quality assessment: sensory and chemical analyses (e.g., liquid chromatography, infrared spectroscopy and mass spectrometry). The sensory analysis mainly detects milk colour, viscosity, smell, flavour and crystallisation. However, the accuracy of the sensory analysis is limited and usually depends on the experience of the sensory panellists. Chemical analysis also has some disadvantages, such as need for highly skilled labour, ultrapure preparation, expensive instruments and large instrumental analysis [1-5]. Thus, the development of a relatively fast, easy-to-use and low-cost measurement method becomes attractive. 
A microwave-based technique can be an alternative method for quality assessment on the basis of the electrical permittivity, which is an intrinsic parameter of a material that represents the interaction with the electromagnetic field. Establishing explicit relation-ships between the permittivity and the agri-food constituents can provide a means of rapid inspection of properties (e.g., moisture, fat and salt content) [6]. Microwave detection methods have various attributes, such as fast (few minutes rather than hours), nonhazardous, capable of measuring bulk properties and less sensitive to environmental conditions [7]. A number of microwave techniques have been reported in the literature and used in practice for liquids: open-ended rectangular waveguide [8]/coaxial probe [9] techniques, time domain spectroscopy/reflectometry (TDR) method [10], resonance methods (e.g., resonant cavity [11] and coupled split-ring resonator [13] and free space methods [14]. Each method has its own particular advantages, disadvantages and applications. Specifically, the open-ended methods are prone to errors introduced by improper contact between the probe and the material surface (e.g., air gaps or air bubbles). The TDR measuring instruments are expensive [12]. The resonance approach is inherently narrowband and requires careful sample preparation and calibration [7]. For the free space technique, special attention must be paid to the choice of horn antennae, design of the sample holder and the sample geometry and location. On the other hand, the transmission line technique is robust, broadband and suited to materials with a wide range of dielectric loss. For the liquid measurement, two waveguide cells with window can be made to hold the sample on each side. However, few studies have been made on the measurement of milk using this technique.

In this work, the transmission line technique is adopted for the study of milks and milk adulteration for the first time. First, the cole-cole equation for the description of dielectric properties of liquids is introduced. Then the liquid permittivity measurement and the available methods for the permittivity calculation are addressed. From the measurement of distilled water at room temperature over two frequency bands (i.e., 6-8 $\mathrm{GHz}$ and 8-12 GHz), the efficiency and accuracy of each permittivity calculation method and frequency band are evaluated by comparison with the empirical data provided by the cole-cole equation. Afterwards, the measurements of two types of milks are conducted over 6-8 GHz. A genetic algorithm, an optimisation method, is employed to quickly search the possible permittivity of the milk samples, which will be a starting point for further accurate calculation. It is also used to obtain the cole-cole dielectric parameters for clear and set milks. The effect of the calibration errors on the accuracy of the permittivity measurement is studied. In addition, its accuracy is compared with that by a commercially available dielectric probe. Finally, the effect of the added water on the permittivity of milk-water mixture is investigated in detail and a purity index is identified.

\section{DIAELECTRIC PROPERTIES OF LIQUIDS}

\subsection{Definition of Permittivity}

When microwave energy is directed towards a material, part of the energy is reflected, part is transmitted and part is absorbed by the sample. The portion of energy that falls into these three categories have been defined in terms of the scattering parameters and these can be related to the dielectric properties of the sample [6]. The fundamental electrical property through which the interactions between the electromagnetic wave and the material are described is the complex permittivity $\varepsilon$. It is mathe-matically expressed as:

$$
\varepsilon 1 / 4 \varepsilon 0 \varepsilon r
$$

Eris the relative permittivity. The real part $\varepsilon 0$ rofer, or di electric constant, characterises the ability of a material or a substance to store the electric field energy. $\varepsilon 00$ r (positive), or dielectric loss factor, reflects the ability of a material to dissipate the electric energy in the form of heat.

The variation of the relative permittivity of water with respect to frequency and temperature over 1e20 $\mathrm{GHz}$ is plotted in Figure 1. In this frequency range, $\varepsilon 0 \mathrm{r}$ and $\varepsilon 00 \mathrm{r}$ are of the same order of magnitude, which demonstrates the high-loss characteristic of water.

\subsection{Penetration Depth of Microwaves into Dielectrics}

Considering the effect of the lossy medium, the power of the microwaves decays exponentially through the thickness. The penetration depth dp is a practical parameter used for the evalua-tion of signal propagation. It is defined as the depth where the amplitude of the signal is reduced to 1/e (about $37 \%$ ) below the surface. For a dielectric material, dp can be calculated by (Pozar, 2011): 


\section{HORN ANTENNAS}

Horn antennas are very popular at UHF (300 MHz-3 GHz) and higher frequencies (I've heard of horn antennas operating as high as $140 \mathrm{GHz}$ ). Horn antennas often have a directional radiation pattern with a high antenna gain, which can range up to $25 \mathrm{~dB}$ in some cases, with 10-20 dB being typical. Horn antennas have a wide impedance bandwidth, implying that the input impedance is slowly varying over a wide frequency range (which also implies low values for S11 or VSWR). The bandwidth for practical horn antennas can be on the order of 20:1 (for instance, operating from $1 \mathrm{GHz}-20 \mathrm{GHz}$ ), with a 10:1 bandwidth not being uncommon.

The gain of horn antennas often increases (and the beam width decreases) as the frequency of operation is increased. This is because the size of the horn aperture is always measured in wavelengths; at higher frequencies the horn antenna is "electrically larger"; this is because a higher frequency has a smaller wavelength. Since the horn antenna has a fixed physical size (say a square aperture of $20 \mathrm{~cm}$ across, for instance), the aperture is more wavelengths across at higher frequencies and a recurring theme in antenna theory is that larger antennas (in terms of wavelengths in size) have higher directivities.

Horn antennas have very little loss, so the directivity of a horn is roughly equal to its gain. Horn antennas are somewhat intuitive and relatively simple to manufacture. In addition, acoustic horn antennas are also used in transmitting sound waves (for example, with a megaphone). Horn antennas are also often used to feed a dish antenna, or as a "standard gain" antenna in measurements.

\section{HORN ANTENNA DESIGNING}

Horns are among the simplest and most widely used microwave antennas and they find applications in the areas of wireless communications, electromagnetic sensing RF heating and biomedicine [15]. The horn antenna may be considered as an RF transformer or impedance match between the waveguide feeder and free space which has an impedance of 377 ohms by having a tapered or having a flared end to the waveguide. Horn antenna offers several benefits when employed in that besides matching the impedance of the guide to that of free space or vice versa, it helps suppress signals travelling via unwanted modes in the waveguide from being radiated and it provides significant level of directivity and gain [16]. While it serves as entry medium for signal interception for processing in the case of receiving systems, it serves in the case of transmission to illuminate dish antenna from its focal area estimated from the f/d parameters of the parabolic dish [17]. Dual mode feed horns often provide excellent performance over wide range of microwave bands [18]. The two basic types of horn antenna are the pyramid and conical horn antenna. Other modifications include sectorial (E or H plane), exponential, corrugated, ridged and septum horns. Figures 1 of pyramidal and conical horns are shown below [16].
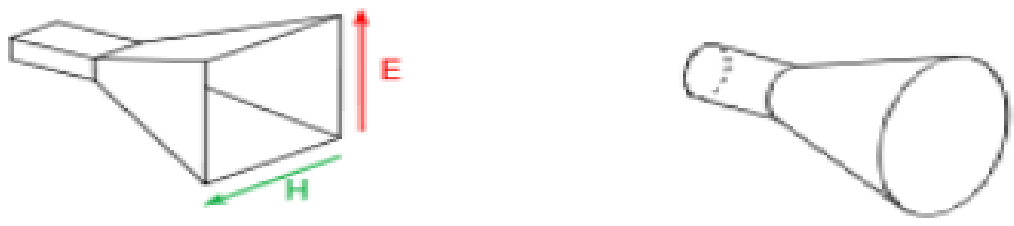

Figure 1. Pyramidal and Conical

Impedance matching is very desirable with radio frequency transmission lines. Standing waves lead to increased losses and frequently cause the transmitted to malfunction [19].

\section{EXPERIMENTAL SETUP}

The set up for the Evaluation of water content in milk using microwave transmission approach with horn antennais shown in Figure 2. The dotted line blocks at the transmitting end are showing the set up for a conventional microwave bench while the solid block indicating the setup presently used in labs. For reception of the RF signal another antenna is placed on a rotating table before the detector. We can use either of three different power detectors. We use Power meter for detection of power. In fact rotating table is not required for this experiment. We are putting milk in between transmitting \& receiving antenna which you can see from the photo of experimental setup as shown in Figure 3. 


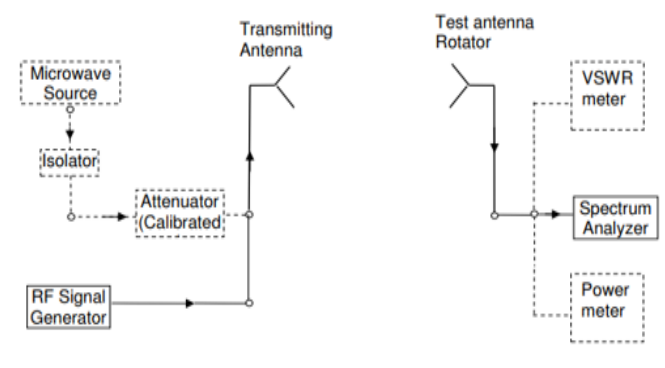

Figure 2. Experimental Setup

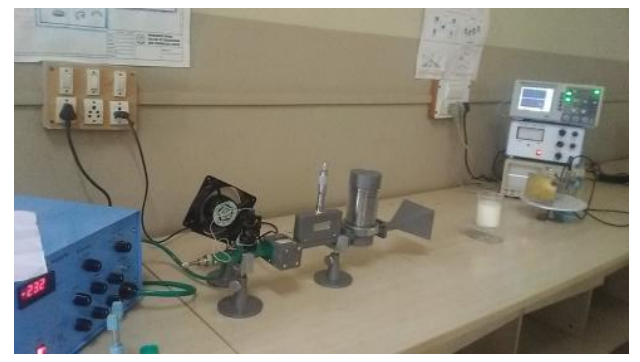

Figure 3. Photo of Experiment Setup

\section{6.}

EXPERIMENTAL RESULTS

The result of the experimental is shown in Table 1.

Table 1. Experimental Results

\begin{tabular}{|c|c|c|c|}
\hline No & Repeller voltage & Beam current & $\begin{array}{c}\text { Vswr } \\
\text { Power in } \mathrm{db}\end{array}$ \\
\hline 1 & 281 & 19.7 & 11.9 \\
\hline 2 & 281 & 19.7 & 20.5 \\
\hline 3 & 281 & 19.7 & 21.2 \\
\hline 4 & 281 & 19.7 & 21.5 \\
\hline 5 & 281 & 19.7 & 12.1 \\
\hline 6 & 281 & 19.7 & 20.06 \\
\hline 7 & 281 & 19.7 & 21.4 \\
\hline 8 & 281 & 19.7 & 21.5 \\
\hline 9 & 282 & 19.7 & 11.8 \\
\hline 10 & 282 & 19.7 & 12.1 \\
\hline 11 & 282 & 19.7 & 21.2 \\
\hline 12 & 282 & 19.7 & 21 \\
\hline
\end{tabular}

\section{EXPERIMENTAL SETUP WITH HORN ANTENNA}

The horn antenna setup is set in the minimum distance by the formula. Us our horn antenna is having the width of $0.1 \mathrm{M}$, It is kept with the minimum distance. We use the $50 \mathrm{ml}$ beaker in which the milk is tested.

At First the the beaker is ket empty and radiated power through the glass is calculated with VSWR meter. Then water is poured in the beaker and then the radiated through the water and received power is calculated. Then $10 \mathrm{ml}, 20 \mathrm{ml}, 30 \mathrm{ml}, 40 \mathrm{ml}, 50 \mathrm{ml}$. The measured values of decibel is plotted in the Table 1.

\section{DISCUSSIONS}

The testing of milk thickness with water proves that the intensity of the milk increases the power of the received decibel is less. By using the microwave horn antenna the milk thickness can be calculated. So we train a nural network system for analysing the thickness of the milk.

\section{NEURAL NETWORK BASED SYSTEM}

Neural network based system is shown in Figure 4, 5, and 6.

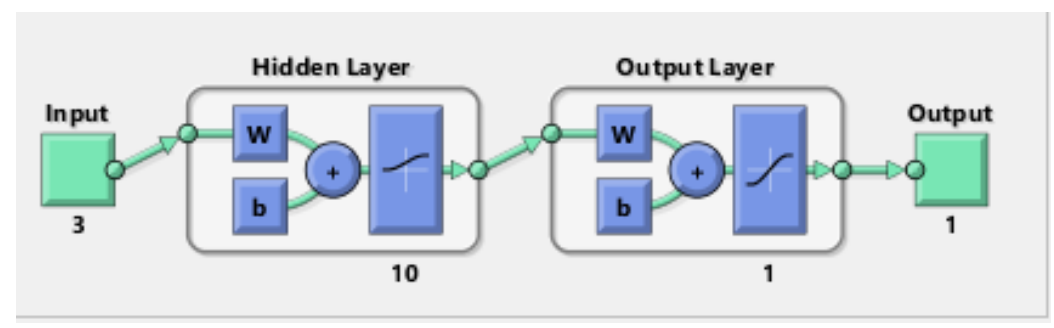

Figur 4. Nural Network 


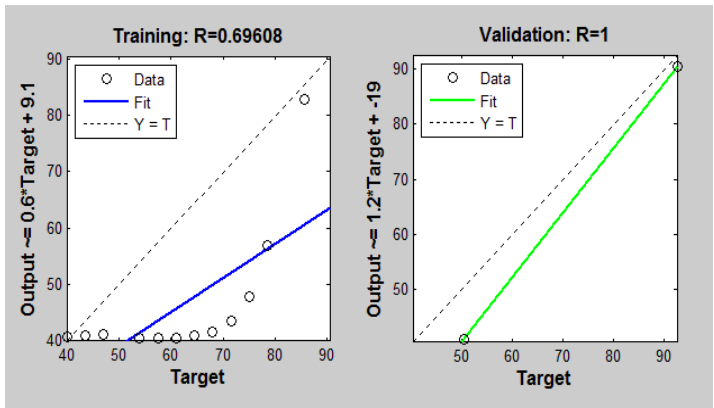

Figure 5. Output of Nural Network

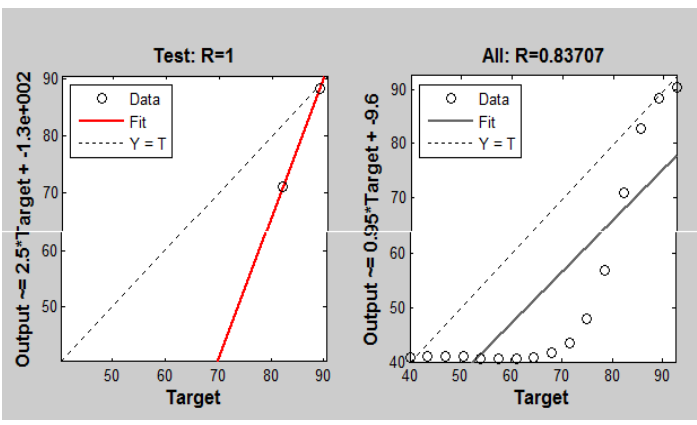

Figure 6. Output of Nural Network

\section{RESULT}

The potential of the microwave transmission line technique for evaluation of added water content in milk has presented. The permittivity can be used as a milk purity indicator. The permittivity of the thick milk is slightly smaller than that of the diluted milk. The permittivity decreases with increasing frequency. From the study of the measurement uncertainty, it has been shown that high accuracy of permittivity calculation can be achieved. By using nural network Evaluation of water content in milk using microwave transmission approach with horn antenna is easy for us to achive.

\section{REFERENCES}

[1] Shiinoki, Y., Motouri, Y., Ito, K., "On-line Monitoring of Moisture and Salt Contents by the Microwave 'lkansmission Method in a Continuous Salted Butter-making Process", Journal of Food Eqheen'ng 38 (1998) 15316701998 Ekevier Science Limited.

[2] Agranovich, D., Renhart, I., Ishai, P.B., Katz, G., Bezman, D., Feldman, Y., "A microwave sensor for the characterization of bovine milk", Food Control 0956-7135/@ 2015 Elsevier Ltd.

[3] The effect of microwave pasteurization onthe composition of milk

[4] Michael O. Fryer, Andrea J. Hills, L. Morrison,"Method and apparatus for measuring butterfat and protein content using microwave absorption techniques" U.S. Patent Nov. 14,2000,US 6147502

[5] Bazar, G., Romv_ari, R., Szab_o, A., Somogyi, T., _Eles, V., Tsenkova, R., 2016. NIR “detection of honey adulteration reveals differences in water spectral pattern."

[6] Gibson, A.A.P., Ng, S.K., Noh, B.B.M., Chua, H.S., Haigh, A.D., Parkinson, G.,Ainsworth, P., Plunkett, A., 2008. "An overview of microwave techniques for theefficient measurement of food materials". Food Manuf. Effic. 2, 1e9.

[7] Kraszewski, A.W., Nelson, S.O., 1992. Observations on resonant cavity perturbationby dielectric objects. IEEE Trans. Microw. Theory Tech. 40, 151e155. http://dx.doi.org/10.1109/22.108334.

[8] Karpisz, T., Kopyt, P., Salski, B., Krupka, J., 2016. Open-ended waveguide measurementof liquids at millimeter wavelengths. In: 2016 IEEE MTT-S International Microwave Symposium (IMS). IEEE, pp. $1 \mathrm{e} 4$. http://dx.doi.org/10.1109/MWSYM.2016.7539954.

[9] Guo, W., Zhu, X., Liu, Y., Zhuang, H., 2010. "Sugar and water contents of honey withdielectric property sensing". J. Food Eng. 97, 275e281. http://dx.doi.org/10.1016/j.jfoodeng.2009.10.024.

[10] Puranik, S., Kumbharkhane, A., Mehrotra, S., 1991. "Dielectric properties of honeywatermixtures between $10 \mathrm{MHz}$ to $10 \mathrm{GHz}$ using time domain technique". J. Microw. Power Electromagn. Energy 26, 196e201. http://dx.doi.org/10.1080/08327823.1991.11688157.

[11] Liao, X., Raghavan, V.G., Meda, V., Yaylayan, V.A., 2001. "Dielectric properties of supersaturated Alpha-D-glucose aqueous solutions at $2450 \mathrm{MHz}$ ". J. Microw. Power

[12] Venkatesh, M.S., Raghavan, G.S.V., 2005. "An overview of dielectric propertiesmeasuring techniques". Can. Biosyst. Eng./Le. Genie Des. Biosyst. au Can. 47,15e30.http://dx.doi.org/10.1109/URSIGASS.2011.6050287.

[13] Watts, C., Hanham, S.M., Ahmad, M.M., Adabi, M., Klein, N., 2016. "Coupled dielectricsplitring microwave resonator for liquid measurements in microfluidic channelsat nanoliter" volumes. In: 46th European Microwave Conference,pp. 257e260.

[14] Jose, K.A., Varadan, V.K., Varadan, V.V., 2001. "Wideband and noncontact characterization of the complex permittivity of liquids". Microw. Opt. Technol. Lett. 30,75e79. http://dx.doi.org/10.1002/mop.1225.

[15] Konstantinos B. Baltzis "Polynomial - Based Evaluation of the Impact of Aperture Phase Taper on the Gain of Rectangular Horns”, Journal of Electromagnetic Analysis \& Applications, 2010, Vol. 2, pp424 - 430

[16] Wikipedia, www.enwikipedia.org/wiki/Horn_antenna.

[17] Dick Comly, "Parabolic Antennas and their Feeds". N3AOG. www. Packratvhf.com/Article 9/Dish_Not.pdf

[18] Paul Wade, "Optimum Dual - mode Feedhorn". W1Ghz, 2006

[19] Samuel Y. Liao, "Microwave Devices and circuits, 3rd Edition”, Prentice Hall, New Jersey, 1990.

[20] Ghanem, T.H "dielectric properties of liquid foods affected by moisture contents and temperatures", Misr J. Ag. Eng., 27 (2): 688 - 698, Process Engineering. 